\title{
Microbiology Specimen Character Result in Standard Format
}

National Cancer Institute

\section{Source}

National Cancer Institute. Microbiology Specimen Character Result in Standard Format. NCI Thesaurus. Code C87900.

The standard character or string for representation and reporting of microbiology specimen data. 